TITLE:

\title{
Nodal superconducting order parameter and thermodynamic phase diagram of (TMTSF)_\{2\}ClO_\{4\}
}

\section{$\operatorname{AUTHOR}(S):$}

Yonezawa, Shingo; Maeno, Yoshiteru; Bechgaard, Klaus; Jérome, Denis

\section{CITATION:}

Yonezawa, Shingo ...[et al]. Nodal superconducting order parameter and thermodynamic phase diagram of (TMTSF)_\{2\}ClO_\{4\}. Physical Review B 2012, 85(14): 140502.

ISSUE DATE:

2012-04

URL:

http://hdl.handle.net/2433/170100

RIGHT:

(C)2012 American Physical Society 


\title{
Nodal superconducting order parameter and thermodynamic phase diagram of (TMTSF $)_{2} \mathrm{ClO}_{4}$
}

\author{
Shingo Yonezawa, ${ }^{1,{ }^{*}}$ Yoshiteru Maeno, ${ }^{1}$ Klaus Bechgaard, ${ }^{2}$ and Denis Jérome ${ }^{3}$ \\ ${ }^{1}$ Department of Physics, Graduate School of Science, Kyoto University, Kyoto 606-8502, Japan \\ ${ }^{2}$ Department of Chemistry, Oersted Institute, Universitetsparken 5, 2100 Copenhagen, Denmark \\ ${ }^{3}$ Laboratoire de Physique des Solides (UMR 8502), Université Paris-Sud, 91405 Orsay, France
}

(Received 31 August 2011; published 3 April 2012)

\begin{abstract}
The organic materials (TMTSF) ${ }_{2} X$ are unique unconventional superconductors with archetypal quasi-onedimensional (Q1D) electronic structures. Here, based on our comprehensive field-angle-resolved calorimetry of (TMTSF $)_{2} \mathrm{ClO}_{4}$, we succeeded in mapping the nodal gap structure by discriminating between the Fermi wave vectors and Fermi velocities. In addition, the thermodynamic phase diagrams of (TMTSF) ${ }_{2} \mathrm{ClO}_{4}$ for all principal field directions are obtained. These findings, providing strong evidence of nodal spin-singlet superconductivity, serves as solid bases for the further elucidation of anomalous superconducting phenomena in (TMTSF) $2 X$.
\end{abstract}

DOI: 10.1103/PhysRevB.85.140502

PACS number(s): 74.20.Mn, 74.25.Bt, 74.25.Dw, 74.70.Kn

In 1980, the first organic superconductor tetramethyltetraselena-fulvalene (TMTSF) salt was discovered. ${ }^{1,2}$ Since then, (TMTSF $)_{2} X\left(X=\mathrm{ClO}_{4}, \mathrm{PF}_{6}\right.$, etc. $)$ have been widely studied because of their interesting properties resulting from their archetypal quasi-one-dimensional (Q1D) conductivity and strong electron-electron interactions. ${ }^{3-6}$ Interestingly, the superconducting (SC) phase is located next to a magnetic phase, resembling superconductivity in other unconventional superconductors such as high- $T_{\mathrm{c}}$ cuprates and pnictides. ${ }^{7,8}$ Because of this similarity, as well as the simplicity of the electronic structure, investigations of (TMTSF $)_{2} X$ can provide useful guidelines for the studies of other unconventional superconductors.

Many studies have revealed unusual SC phenomena in $(\mathrm{TMTSF})_{2} X$. For example, the onset of superconductivity in resistivity is observed even above $4 \mathrm{~T}$ when the field is parallel to the $a$ axis, the most conducting direction, or to the $b^{\prime}$ axis, the second-most conducting direction. ${ }^{9-12}$ This fact indicates that a certain contribution of superconductivity survives beyond the Pauli-limiting field $\mu_{0} H_{\mathrm{P}} \sim 2.3-2.6 \mathrm{~T}$, ${ }^{12}$ where ordinary singlet pairs would be unstable due to the Zeeman splitting. Thus, the possibilities of a spin-triplet pairing state or a spatially modulated spin-singlet pairing state, which is the so-called Fulde-Ferrell-Larkin-Ovchinnikov (FFLO) state, have been discussed. $^{9-14}$ In addition, the high-field superconductivity is accompanied by a peculiar anisotropy of the resistivity onset. ${ }^{11,12}$ However, to the best of our knowledge, SC phase diagrams have not been established from thermodynamic measurements.

For clarification of the origin of such unusual behavior, as well as for identification of the SC mechanism of (TMTSF) $)_{2} X$, orbital and spin parts of the SC order parameter are essentially important. Although sign changes on the SC gap are evidenced by the suppression of superconductivity by a small amount of nonmagnetic impurities, ${ }^{15,16}$ details of the gap structure and the SC symmetry are still controversial. ${ }^{17-19}$ Theories based on a simple Q1D model with the Fermi surface (FS) consisting of a pair of warped sheets have revealed that a spin-singlet $d$ wave-like state with line nodes is stable when spin fluctuations drive the pairing, ${ }^{4,20}$ whereas a spin-triplet $f$-wave-like state with similar line nodes can be stable when charge fluctuations are incorporated. ${ }^{4}$ The FS of the $\mathrm{ClO}_{4}$ salt is slightly different from the simple model due to the orientational order of the tetrahedral $\mathrm{ClO}_{4}$ anions at $T_{\mathrm{AO}}=24 \mathrm{~K}$; this order leads to a folding of the band structure along the $b^{*}$ direction and the FS splits into two pairs. ${ }^{21}$ A nodeless $d$-wave-like, a nodeless $f$ wave-like, and a nodal $d$-wave-like states have been proposed for such a FS. ${ }^{22,23}$

To clarify these issues, we performed field-angle-resolved calorimetry for one piece of a (TMTSF) ${ }_{2} \mathrm{ClO}_{4}$ single crystal. We used a crystal weighing as low as $76 \mu \mathrm{g}$ grown by an electrocrystallization technique. ${ }^{24}$ This piece of crystal was used in our previous transport study, and was confirmed to be very clean with a mean free path as large as $1.6 \mu \mathrm{m} .^{11,12} \mathrm{We}$ used a ${ }^{3} \mathrm{He}-{ }^{4} \mathrm{He}$ dilution refrigerator to cool the sample. After we cooled the cryostat and the sample to $4.2 \mathrm{~K}$, we heated the sample again to $26 \mathrm{~K}$, and cooled it very slowly across $T_{\mathrm{AO}}$ to $20 \mathrm{~K}$ at $4 \mathrm{mK} / \mathrm{min}$, so that the anions order well to be in the "relaxed" state. We developed a high-resolution $(\sim 100 \mathrm{pJ} / \mathrm{K}$ at $1 \mathrm{~K}$ ) calorimeter shown in Fig. 1(b), based on a modification of the "bath-modulating method." 25 The advantage of this technique is that a heater on the sample holder is not necessary; thus the background heat capacity of the sample holder can be minimized. Although this background contribution is not subtracted from the data shown here, we have checked that the background is nearly field independent. ${ }^{24}$ The magnetic field is applied using the vector magnet system. ${ }^{26}$ The magnetic field is aligned to the crystalline axes by making use of the anisotropy in the upper critical field $H_{\mathrm{c} 2}$. The precision and accuracy of the field alignment is approximately $0.1^{\circ}$. More details of the experimental procedure will be described elsewhere. ${ }^{27}$

First we focus on the field dependence of $C / T$ plotted in Fig. 1. For $H \| c^{*}$, i.e., fields perpendicular to the conducting $a b^{\prime}$ plane, $C(H) / T$ exhibit a $H^{0.5}$ dependence at low temperatures. This dependence provides strong evidence for a line-node gap. ${ }^{28}$ In contrast, $C(H) / T$ for $H \| a$, for which the orbital pair breaking is substantially weakened, exhibits a concave-up curvature at low temperatures [Fig. 1(a)] near $H_{\mathrm{c} 2}$. Considering the clear change of the spin susceptibility in the SC state, ${ }^{18}$ we identify this behavior as the Pauli-limiting behavior in a spin-singlet superconductor. ${ }^{29}$

To investigate the nodal structure of the SC gap, we measured the in-plane field-angle dependence of $C / T$ represented in Figs. 2 and 3. When the SC gap has a node or a zero 
(TMTSF) $)_{2} \mathrm{ClO}_{4}+$ background; $0.11 \mathrm{~K}$
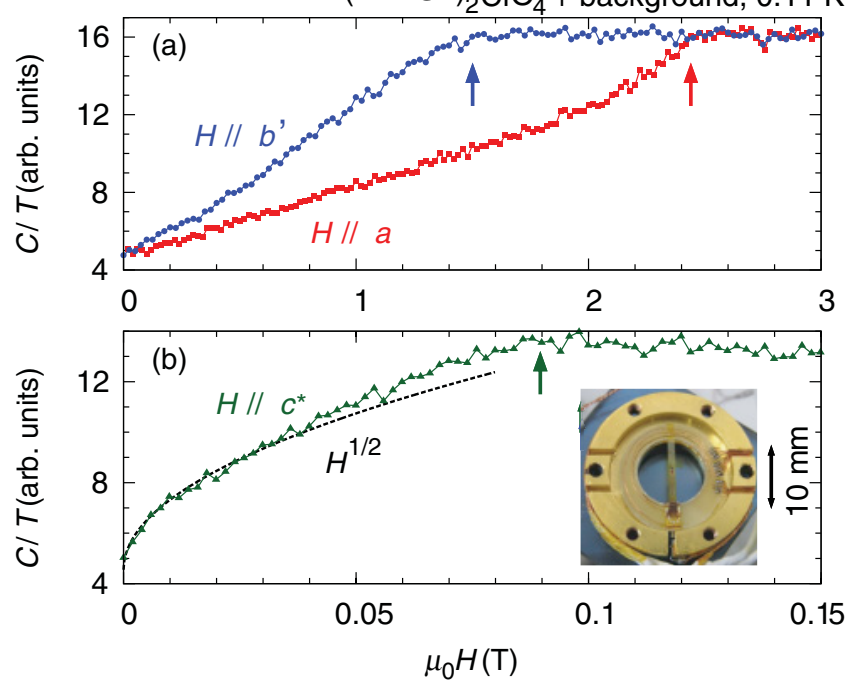

FIG. 1. (Color online) Magnetic field dependence of $C / T$ at $0.11 \mathrm{~K}$ for (a) $H \| a$ (red/gray squares), $H \| b^{\prime}$ (blue/dark gray circles), and (c) $H \| c^{*}$. The arrows indicate the onset $H_{\mathrm{c} 2}$. The broken curve in (b) illustrates $H^{0.5}$ behavior. The inset in (b) is a photo of our calorimeter.

at $\boldsymbol{k}_{\mathrm{F}}^{\text {node }}$, the quasiparticle (QP) density of states (QDOS) $N$, which is proportional to $C_{e} / T$ at low temperatures, varies with the field direction. Here, $C_{e}$ is the electronic heat capacity. In the low-temperature and low-field limit, such a variation of QDOS originates from the "Doppler shift" of the QP energy $\delta \omega(\boldsymbol{r}, \boldsymbol{k}) \propto \boldsymbol{v}_{\mathrm{s}}(\boldsymbol{r}) \cdot \boldsymbol{v}_{\mathrm{F}}(\boldsymbol{k})$, where $\boldsymbol{v}_{\mathrm{s}}(\boldsymbol{r})$ is the velocity of the supercurrent around a vortex and $\boldsymbol{v}_{\mathrm{F}}(\boldsymbol{k})$ is the Fermi velocity. ${ }^{28,30}$ When $\delta \omega$ is larger than the gap $\Delta(\boldsymbol{k})$, QPs with the wave vector $\boldsymbol{k}$ are excited. Because most of the excitation occurs in the vicinity of the nodes, the most important term is $\delta \omega^{\text {node }} \propto \boldsymbol{v}_{\mathrm{s}} \cdot \boldsymbol{v}_{\mathrm{F}}^{\text {node }}$, where $\boldsymbol{v}_{\mathrm{F}}^{\text {node }} \equiv \boldsymbol{v}_{\mathrm{F}}\left(\boldsymbol{k}_{\mathrm{F}}^{\text {node }}\right)$. When $\boldsymbol{H}$ is parallel to $\boldsymbol{v}_{\mathrm{F}}^{\text {node }}, \delta \omega^{\text {node }}$ becomes zero (i.e., $\boldsymbol{v}_{\mathrm{s}} \perp \boldsymbol{v}_{\mathrm{F}}^{\text {node }}$ ) because $\boldsymbol{v}_{\mathrm{s}}$ is perpendicular to $\boldsymbol{H}$, and QDOS induced by this shift becomes small [Fig. 4(a)]. Using this idea, we can investigate the directions of $\boldsymbol{v}_{\mathrm{F}}$ at nodal positions. If the condition $\boldsymbol{k}_{\mathrm{F}} \| \boldsymbol{v}_{\mathrm{F}}$ is satisfied, the $k$-space nodal direction equals the field direction for which $C / T$ exhibits minimum. ${ }^{31}$ For quasi-two-dimensional (Q2D) or three-dimensional (3D) systems, the assumption $\boldsymbol{k}_{\mathrm{F}} \| \boldsymbol{v}_{\mathrm{F}}$ is reasonable as a simple model. In contrast, for Q1D systems, $\boldsymbol{v}_{\mathrm{F}}$ is not parallel to $\boldsymbol{k}_{\mathrm{F}}$, even in the simplest model. Thus, to deduce the nodal position in the $k$ space, information about the band structure is required. Another difficulty for Q1D systems is that a large in-plane anisotropy in $H_{\mathrm{c} 2}$ also contributes to the anisotropy in $C_{e}$. Because of these difficulties, $C_{e} / T$ oscillation in Q1D systems has been little studied, despite its essential importance in determining the SC gap structure.

Interestingly, we find that the $C(\phi) / T$ curves of (TMTSF) ${ }_{2} \mathrm{ClO}_{4}$, where $\phi$ is the azimuthal angle of the field measured from the $a$ axis, become asymmetric with respect to the $a$ axis [e.g., $C(\phi)>C(-\phi)$ for $0^{\circ}<\phi<90^{\circ}$ ] at low temperatures and low fields, as shown in Figs. 2(a) and 2(b). In contrast, the curves are nearly symmetric at high temperatures or in high fields. Thus the asymmetry is not due to a misalignment of the field. What is more, at $0.14 \mathrm{~K}$, small kinks
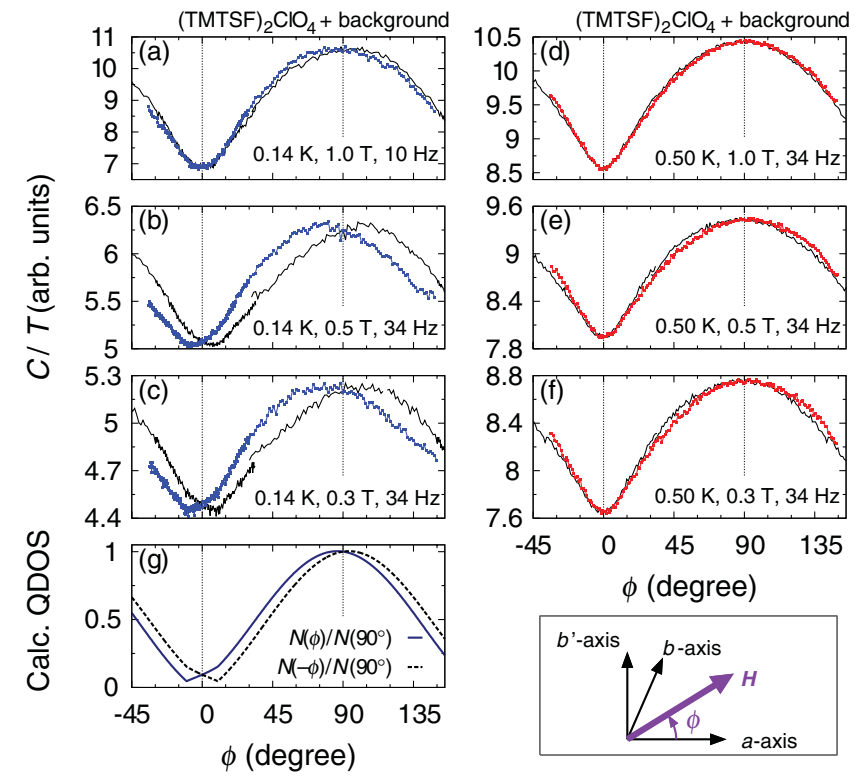

FIG. 2. (Color online) Magnetic-field-angle $\phi$ dependence of the heat capacity for fields rotated within the conducting $a b^{\prime}$ plane at (a)-(c) $0.14 \mathrm{~K}$ and (d)-(f) $0.50 \mathrm{~K}$. For comparison, the same data are also plotted against $-\phi$ with appropriate shifting (the black curves). The deviation of the two curves indicates the asymmetry in the $C(\phi) / T$ curve. (g) QDOS $N(\phi) / N\left(90^{\circ}\right.$ ) (blue solid curve) and $N(-\phi) / N\left(90^{\circ}\right)$ (black dotted curve) calculated by Eq. (1) assuming two nodes (n1 and n2) with the parameters $\phi_{\mathrm{n} 1}=-10^{\circ}$ and $\phi_{\mathrm{n} 2}=+10^{\circ}, A_{\mathrm{n} 2} / A_{\mathrm{n} 1}=0.3$, and $\Gamma=H_{\mathrm{c} 2}\left(0^{\circ}\right) /$ $H_{\mathrm{c} 2}\left(90^{\circ}\right)=3.5$.

are observed at around $\phi= \pm 10^{\circ}$, as shown in Fig. 3(a). The kink signatures are more obvious in the derivatives [Fig. 3(b)]: The steplike behavior in the first derivative and the peaks in the second derivative provide clear indication of the kinks.

The results qualitatively agree with the theoretical expectation that the specific-heat anomaly due to the gap anisotropy should appear only in the low-temperature and low-field region. ${ }^{31,32}$ Thus we attribute them to the SC gap anisotropy. Below, we demonstrate that a simple model based on the Doppler shift reproduces the key features of the unconventional behavior. In order to incorporate the large in-plane $H_{\mathrm{c} 2}$ anisotropy into the Doppler-shift mechanism, ${ }^{30}$ we assume that QDOS $N(\phi)$ for $H \ll H_{\mathrm{c} 2}(\phi)$ varies as $N(\phi) \propto$ $\sqrt{H / H_{\mathrm{c} 2}(\phi)} \sum_{n} A_{n}\left|\sin \left(\phi-\phi_{n}\right)\right|$, where $\phi_{n}$ is the direction of $\boldsymbol{v}_{\mathrm{F}}$ at the $n$th node/zero. To take into account the triclinic band structure, we introduce the nodal-position-dependent amplitude $A_{n}$. We approximate $H_{\mathrm{c} 2}(\phi)$ to follow the effective mass model $H_{\mathrm{c} 2}(\phi)=H_{\mathrm{c} 2}\left(0^{\circ}\right) /\left(\Gamma^{2} \sin ^{2} \phi+\cos ^{2} \phi\right)^{1 / 2}$, with $\Gamma \equiv H_{\mathrm{c} 2}\left(0^{\circ}\right) / H_{\mathrm{c} 2}\left(90^{\circ}\right)$. Thus $N(\phi)$, which is proportional to $C_{e} / T$, should vary as

$$
\begin{aligned}
N(\phi) \propto & \left(\Gamma^{2} \sin ^{2} \phi+\cos ^{2} \phi\right)^{1 / 4} \\
& \times \sqrt{\frac{H}{H_{\mathrm{c} 2}\left(0^{\circ}\right)}} \sum_{n: \text { nodes } / \text { zeros }} A_{n}\left|\sin \left(\phi-\phi_{n}\right)\right| .
\end{aligned}
$$

The calculated $N(\phi)$ with $\phi_{n}= \pm 10^{\circ}$ plotted in Figs. 2(g) and 3(c) captures well the observed unconventional behavior, though we could not perfectly fit the equation to the data. For a better fitting, additional contributions of vortex cores, thermal 


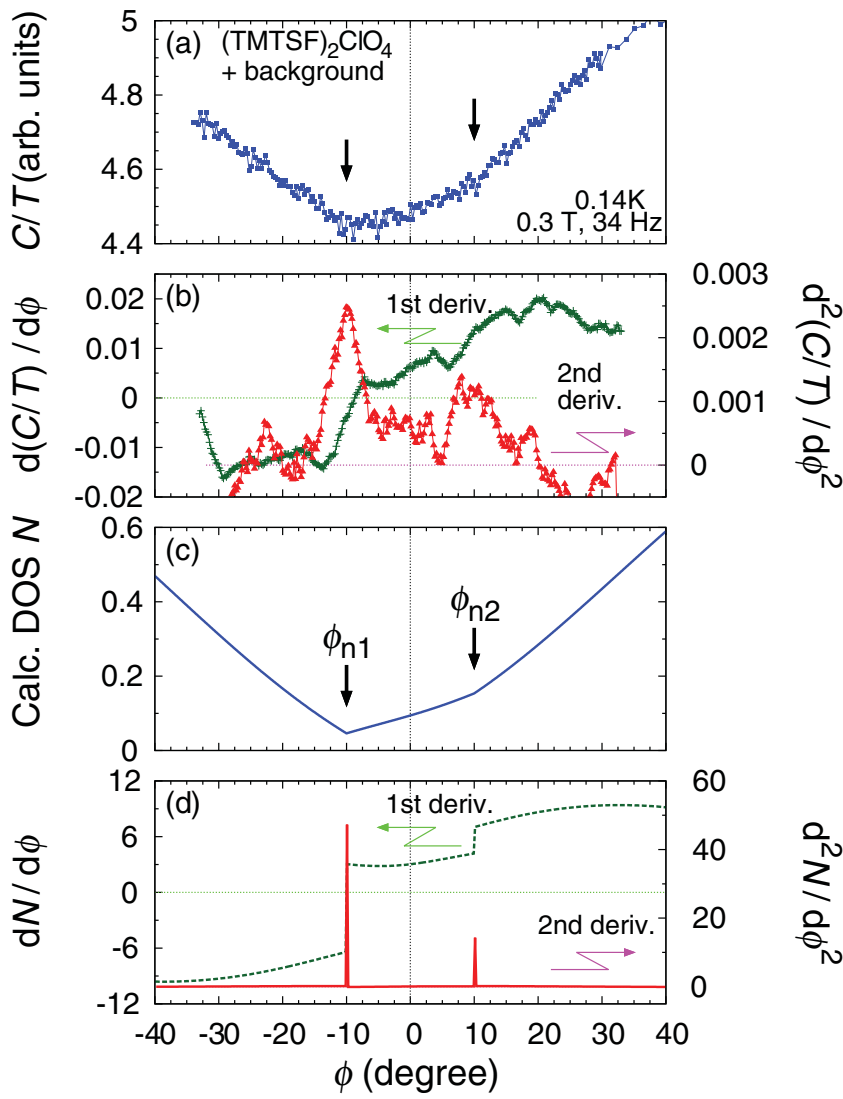

FIG. 3. (Color online) (a) Enlarged view of $C(\phi) / T$ at $0.14 \mathrm{~K}$ and $0.3 \mathrm{~T}$. The arrows indicate the positions of the small kinks. (b) Derivatives $\mathrm{d}(C / T) / \mathrm{d} \phi$ (green crosses) and $\mathrm{d}^{2}(C / T) / \mathrm{d} \phi^{2}$ (red triangles). (c) Calculated DOS near $\phi=0$. (d) Qualitative behavior of $\mathrm{d} N / \mathrm{d} \phi$ (green dotted curve) and $\mathrm{d}^{2} N / \mathrm{d} \phi^{2}$ (red solid curve).

excitations, and the Pauli effect should probably be taken into account. It is worth noting that the behavior of the derivatives is also consistent with the experiment. We note that a recent theory ${ }^{32}$ also attributed the observed calorimetric behavior to the existence of the gap node.

The above analysis indicates that there must be at least a node (or zero) with $v_{\mathrm{F}}$ pointing $\phi=+10^{\circ}$ and another one with $\boldsymbol{v}_{\mathrm{F}}$ pointing $-10^{\circ}$. To determine the nodal position in the $k$ space, we plot in Fig. 4(b) the $k_{y}$ dependence of the velocity angle measured from the $a$ axis, $\phi_{v_{\mathrm{F}}}$, based on the tight-binding band structure. ${ }^{6,21}$ Figure 4(b) manifests that $\left|\phi_{\boldsymbol{v}_{\mathrm{F}}}\right|$ reaches $10^{\circ}$ on the outer FS at $k_{y} \sim \pm 0.25 b^{*},+0.36 b^{*},-0.06 b^{*}$, where $b^{*}$ is the size of the first Brillouin zone along $k_{y}$. Thus, at least, some of the gap nodes or zeros should be located around these positions. The simplest structure that satisfies this condition is a structure with nodes or zeros running at $k_{y}= \pm 0.25 b^{*}$, which is presented in Fig. 4(c). Leading candidates of the pairing state that satisfies the obtained gap structure are the $d$-wave-like or the $g$-wave-like states shown in Fig. 4(c).

Finally, in Fig. 5, we present the thermodynamic SC phase diagrams based on the calorimetry compared with transport phase diagrams based on the $c^{*}$-axis resistivity. ${ }^{11,12}$ The thermodynamic upper critical field $\mu_{0} H_{\mathrm{c} 2}^{\mathrm{thm}}(0) \simeq 2.5 \mathrm{~T}$ for $H \| a$ is much smaller than $\mu_{0} H_{\mathrm{c} 2}^{\text {orb }}=-0.73 T_{\mathrm{c}}\left(\mu_{0} \mathrm{~d} H_{\mathrm{c} 2} /\left.\mathrm{d} T\right|_{T=T_{\mathrm{c}}}\right) \sim$ $7.7 \mathrm{~T}$, which is expected for the orbital pair breaking, but (a)
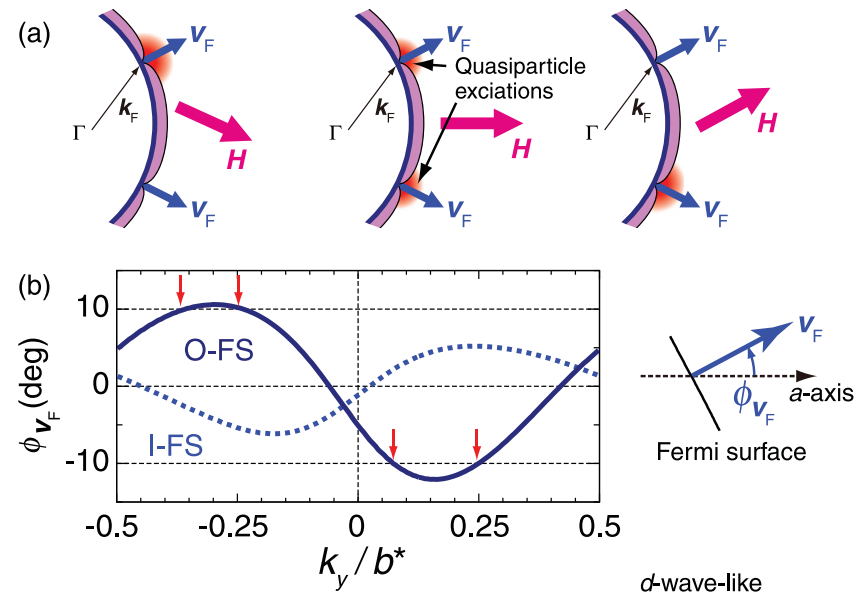

(c)
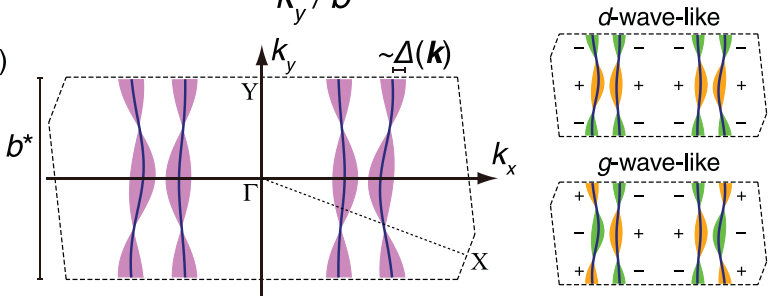

FIG. 4. (Color online) (a) Quasiparticle excitation due to the Doppler shift. When the field is parallel to $\boldsymbol{v}_{\mathrm{F}}$ at a node, the quasiparticle excitation at this node is reduced. (b) Dependence of $\phi_{v_{\mathrm{F}}} \equiv \arctan \left(v_{y} / v_{x}\right)$ on $k_{y}$ for the outer FS (O-FS; solid curve) and inner FS (I-FS; dotted curve). The arrows indicate the points where $\phi_{v_{\mathrm{F}}}$ reaches $\pm 10^{\circ}$. (c) Most plausible gap structure with nodes or zeros at $k_{y} \sim \pm 0.25 b^{*}$. Examples of the superconducting states that satisfy the observed nodal structure are shown: the $d$-wave-like state and the $g$-wave-like state.

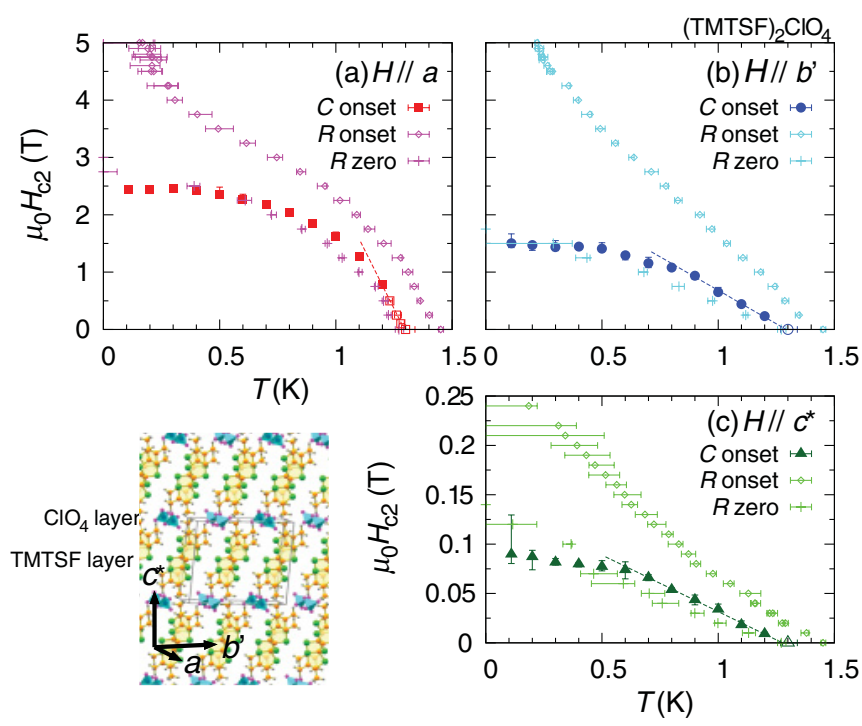

FIG. 5. (Color online) Thermodynamic SC phase diagrams of (TMTSF) ${ }_{2} \mathrm{ClO}_{4}$ compared with the transport phase diagrams for fields parallel to (a) the $a$ axis, (b) the $b^{\prime}$ axis, and (c) the $c^{*}$ axis. The solid symbols are obtained from field sweeps and the open symbols from temperature sweeps. For comparison, we also present the onset temperature of the $c^{*}$-axis resistance $R_{c^{*}}$ (open diamonds), as well as the temperature where $R_{c^{*}}$ becomes zero (crosses), reported in Ref. 11 . The dotted lines indicate the slope $\mu_{0} \mathrm{~d} H_{\mathrm{c} 2}(T) / \mathrm{d} T$ near $T=T_{\mathrm{c}}:-8.1$ $\mathrm{T} / \mathrm{K}$ for $H \| a,-2.3 \mathrm{~T} / \mathrm{K}$ for $H \| b^{\prime}$, and $-0.11 \mathrm{~T} / \mathrm{K}$ for $H \| c^{*}$. The crystalline structure and axes are schematically shown. 
agrees with the Pauli-limiting field $\mu_{0} H_{\mathrm{P}} \sim 2.3-2.6 \mathrm{~T}^{12}$ This fact again supports a spin-singlet scenario. Furthermore, the absence of multiple SC phases provides proof of a singlet state in the whole region below $H_{\mathrm{c} 2}^{\text {thm }}$. Looking again at earlier thermodynamic studies here, one notices that the present $H_{\mathrm{c} 2}^{\text {thm }}(0)$ agrees with the field at which the nuclear-lattice relaxation rate recovers to the normal-state value, ${ }^{18}$ or with the irreversible field in the torque measurement. ${ }^{10}$ Thus, these anomalies reported earlier are now turned out to be due to the thermodynamic SC transition.

Although we used an identical crystal in both the thermodynamic and transport studies, the phase diagrams obtained are quite different. It is clear that a long-range-ordered SC state does not exist above $H_{\mathrm{c} 2}^{\mathrm{thm}}$. In the region between $H_{\mathrm{c} 2}^{\mathrm{thm}}$ and the resistance onset, however, the observed sharp resistance drop and the anisotropy in the onset temperature reflecting the bulk Fermi-surface anisotropy ${ }^{11,12}$ evidence that intrinsic superconductivity robustly contributes to the transport. The superconductivity above $H_{\mathrm{c} 2}^{\mathrm{thm}}$ should be realized without any noticeable entropy change compared to the normal state. Thus, it must be characterized either by a fluctuating order parameter or by a static order parameter accompanied by only a slight change in the density of states. The true nature of the superconductivity in the high-field region is one of the most intriguing problems to be clarified in the future.
In summary, our precise field-angle-resolved calorimetry, using a very clean single crystal of (TMTSF) ${ }_{2} \mathrm{ClO}_{4}$, provides strong evidence of a spin-singlet nodal superconductivity. Our result marks the calorimetric mapping of the gap structure of a Q1D superconductor beyond the constraint previously believed; this technique is indeed applicable regardless of crystalline and electronic symmetries. We also revealed the thermodynamic SC phase diagram, which exhibits substantial deviation from the resistivity onset. The present findings should serve as solid bases for the investigation of the pairing mechanism of (TMTSF) $)_{2} X$, as well as for elucidation of unconventional SC phenomena in this compound.

We acknowledge P. Auban-Senzier, S. Kittaka, and T. Nakamura for their support, and Y. Matsuda, Y. Nagai, R. Ikeda, C. Bourbonnais, Y. Fuseya, A. Kobayashi, Y. Suzumura, A. Schofield, S. Brown, H. Shimahara, and H. Aizawa for useful discussions. We also acknowledge KOA Corporation, TORAY Advanced Film, and TORAY for providing us with their products for the calorimeter. This work is supported by a Grant-in-Aid for the Global COE "The Next Generation of Physics, Spun from Universality and Emergence" and by Grants-in-Aids for Scientific Research (KAKENHI 21110516, 21740253, 23540407, and 23110715) from MEXT and JSPS. *yonezawa@scphys.kyoto-u.ac.jp

${ }^{1}$ D. Jérome, A. Mazaud, M. Ribault, and K. Bechgaard, J. Phys. Lett. 41, L95 (1980).

${ }^{2}$ K. Bechgaard, K. Carneiro, M. Olsen, F. B. Rasmussen, and C. S. Jacobsen, Phys. Rev. Lett. 46, 852 (1981).

${ }^{3}$ T. Ishiguro, K. Yamaji, and G. Saito, Organic Superconductors, 2nd edition (Springer, Heidelberg, 1998).

${ }^{4}$ K. Kuroki and Y. Tanaka, J. Phys. Soc. Jpn. 74, 1694 (2005).

${ }^{5}$ The Physics of Organic Superconductors and Conductors, edited by A. Lebed (Springer, Heidelberg, 2008).

${ }^{6} \mathrm{We}$ note here that a single-electron band picture has been proved to be quite reasonable for (TMTSF) $)_{2} X$ (Refs. 3 and 5) in spite of strong electron-electron correlation. This is in clear contrast to, e.g., $f$-electron heavy-fermion systems.

${ }^{7}$ N. Doiron-Leyraud, P. Auban-Senzier, S. Rene de Cotret, C. Bourbonnais, D. Jérome, K. Bechgaard, and L. Taillefer, Phys. Rev. B 80, 214531 (2009).

${ }^{8}$ K. Jin, N. P. Butch, K. Kirshenbaum, J. Paglione, and R. L. Greene, Nature (London) 476, 73 (2011).

${ }^{9}$ I. J. Lee, M. J. Naughton, G. M. Danner, and P. M. Chaikin, Phys. Rev. Lett. 78, 3555 (1997).

${ }^{10}$ J. I. Oh and M. J. Naughton, Phys. Rev. Lett. 92, 067001 (2004).

${ }^{11}$ S. Yonezawa, S. Kusaba, Y. Maeno, P. Auban-Senzier, C. Pasquier, K. Bechgaard, and D. Jérome, Phys. Rev. Lett. 100, 117002 (2008).

${ }^{12}$ S. Yonezawa, S. Kusaba, Y. Maeno, P. Auban-Senzier, C. Pasquier, and D. Jérome, J. Phys. Soc. Jpn. 77, 054712 (2008).

${ }^{13}$ A. G. Lebed, JETP Lett. 44, 114 (1986).

${ }^{14}$ A. G. Lebed, Phys. Rev. Lett. 107, 087004 (2011).

${ }^{15}$ M. Y. Choi, P. M. Chaikin, S. Z. Huang, P. Haen, E. M. Engler, and R. L. Greene, Phys. Rev. B 25, 6208 (1982).
${ }^{16}$ N. Joo, P. Auban-Senzier, C. R. Pasquier, D. Jérome, and K. Bechgaard, Europhys. Lett. 72, 645 (2005).

${ }^{17}$ M. Takigawa, H. Yasuoka, and G. Saito, J. Phys. Soc. Jpn. 56, 873 (1987).

${ }^{18}$ J. Shinagawa, Y. Kurosaki, F. Zhang, C. Parker, S. E. Brown, D. Jérome, K. Bechgaard, and J. B. Christensen, Phys. Rev. Lett. 98, 147002 (2007).

${ }^{19}$ S. Belin and K. Behnia, Phys. Rev. Lett. 79, 2125 (1997).

${ }^{20}$ R. Duprat and C. Bourbonnais, Eur. Phys. J. B 21, 219 (2001).

${ }^{21}$ D. L. Pevelen, J. Gaultier, Y. Barrans, D. Chasseau, F. Castet, and L. Ducasse, Eur. Phys. J. B 19, 363 (2001).

${ }^{22}$ H. Shimahara, Phys. Rev. B 61, R14936 (2000).

${ }^{23}$ Y. Mizuno, A. Kobayashi, and Y. Suzumura, Physica C 471, 49 (2011).

${ }^{24}$ See Supplemental Material at http://link.aps.org/supplemental/ 10.1103/PhysRevB.85.140502 for information on the sample and the background contribution.

${ }^{25}$ J. E. Graebner, Rev. Sci. Instrum. 60, 1123 (1989).

${ }^{26}$ K. Deguchi, T. Ishiguro, and Y. Maeno, Rev. Sci. Instrum. 75, 1188 (2004).

${ }^{27}$ S. Yonezawa et al. (unpublished).

${ }^{28}$ G. E. Volovik, JETP Lett. 58, 469 (1993).

${ }^{29}$ M. Ichioka and K. Machida, Phys. Rev. B 76, 064502 (2007).

${ }^{30}$ I. Vekhter, P. J. Hirschfeld, J. P. Carbotte, and E. J. Nicol, Phys. Rev. B 59, R9023 (1999).

${ }^{31}$ T. Sakakibara, A. Yamada, J. Custers, K. Yano, T. Tayama, H. Aoki, and K. Machida, J. Phys. Soc. Jpn. 76, 051004 (2007).

${ }^{32}$ Y. Nagai, H. Nakamura, and M. Machida, Phys. Rev. B 83, 104523 (2011). 